in prediction accuracy between constructs was further assessed as integrated discrimination improvement (IDI). Similar AUC and IDI constructs evaluated the transition to obstructive or extensive atherosclerosis at follow-up in patients with baseline non-extensive or non-obstructive disease.

Results: High hs-cTnl (>1.5pg/ml) added to FRS-DA increased AUC from 0.717 to 0.731 (Figure $1 \mathrm{~A}$ ) and improved prediction accuracy for baseline plaque $[\mid \mathrm{DI}=0.041(\mathrm{SE})=0.017, \mathrm{p}=0.015]$. In contrast, a-b2GPI-IgA did not [IDI=0.005 (0.006), $p=0.47]$ and the combination offered no added benefit to the hs-cTnl model alone. Similar observations were made for CAC. Presence of a-b2G$\mathrm{PI}-\lg \mathrm{A}$ independently associated with coronary plaque progression (IRR=1.67 [95\% Cl 1.04-2.67]), whereas hs-cTnl did not. Likewise, a-b2GPI-lgA associated with transition to extensive or obstructive disease independently of FRS-DA (OR=13.48 [95\% Cl 2.09-86.99]). Notably, 71.4\% of a-b2GPI-IgA positive patients with high hs-cTnl progressed to extensive or obstructive disease compared to $7.7 \%$ of a-b2GPI-IgA negative subjects with high hs-cTnl $(p=0.008)$. Addition of a-b2GPI-IgA to FRS-DA in patients with prevalent non-extensive non-obstructive plaque increased AUC from 0.785 to 0.900 (Figure 1B) and significantly improved the prediction for development of obstructive or extensive atherosclerosis at follow-up [0.387, (0.13), $\mathrm{p}=0.003]$

A
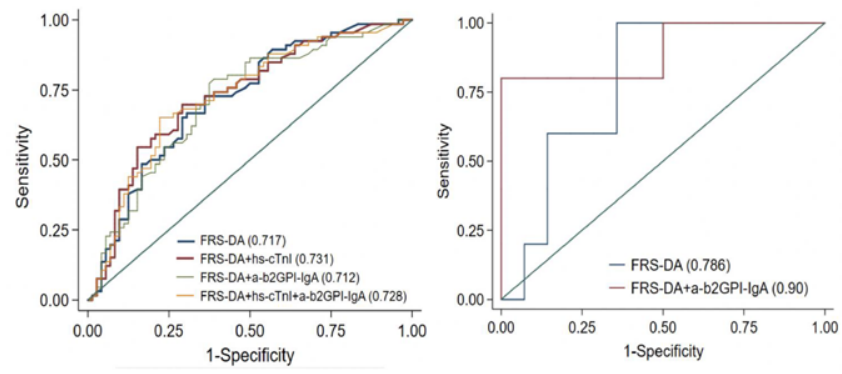

Figure 1. (A) Diagnostic accuracy for prediction of occult coronary atherosclerosis at baseline. FRS-DA alone is the base model followed by addition of hs-cTnl or a-b2GPI-lgA individually or combined. (B) Diagnostic accuracy for progression from non-obstructive and non-extensive plaque at baseline to obstructive or extensive atherosclerosis at follow-up.

Conclusion: High hs-cTnl improved the risk of baseline plaque presence beyond clinical risk score and may trigger an initial non-invasive coronary atherosclerosis evaluation. A-b2GPI-IgA presence may justify a follow-up evaluation in patients with non-extensive, non-obstructive plaque at baseline.

Disclosure of Interests: George Karpouzas Grant/research support from: Pfizer, Consultant of: Sanofi-Genzyme-Regeneron, Janssen, Speakers bureau: Sanofi-Genzyme-Regeneron, BMS, Sarah Ormseth: None declared, Elizabeth Hernandez: None declared, Matthew Budoff: None declared DOI: 10.1136/annrheumdis-2020-eular.5037

\section{THU0531 A PROSPECTIVE STUDY EVALUATING THE ROLE OF 68GA-RGD2 PET/CT ANGIOGENESIS IMAGING IN ASSESSING DISEASE ACTIVITY AND TREATMENT RESPONSE IN RHEUMATOID ARTHRITIS AND ITS COMPARISON WITH DAS28}

A. J. Kavanal ${ }^{1}$, A. Bhattacharya ${ }^{1}$, A. Sharma ${ }^{2}$, J. Shukla ${ }^{1}$, A. Chattopadhyay ${ }^{2}$, R. Vatsa ${ }^{1}$, N. Rana ${ }^{1}$, G. Kaur ${ }^{1}$, B. R. Mittal ${ }^{1} .{ }^{1} P G I M E R$, Nuclear Medicine, Chandigarh, India; ${ }^{2} P G I M E R$, Internal Medicine, Chandigarh, India

Background: PET/CT imaging of synovial angiogenesis using ${ }^{68} \mathrm{Ga}-\mathrm{RGD}$ (cyclic tripeptide agent targeting $a_{v} \beta_{3}$ integrin) to study disease activity in rheumatoid arthritis (RA) has been demonstrated earlier in a few patients. However, post treatment changes in disease activity on ${ }^{68} \mathrm{Ga}-\mathrm{RGD}, \mathrm{PET} / \mathrm{CT}$ imaging have not been adequately assessed.

Objectives: To compare the performance of ${ }^{68} \mathrm{Ga}-\mathrm{RGD}_{2}$ PET/CT with disease activity score (DAS) 28 in assessing disease activity and treatment response in RA.

Methods: Thirty patients $(24 \mathrm{~F}, 6 \mathrm{M})$ aged $43 \pm 12$ years with clinically diagnosed RA were prospectively studied. After calculation of DAS28 by a rheumatologist, all 30 patients underwent ${ }^{68} \mathrm{Ga}-\mathrm{RGD}$, PET/CT scan. Of these, 27 patients underwent a second ${ }^{68} \mathrm{Ga}-\mathrm{RGD}_{2}$ PET/CT scan and clinical assessment after at least 3 months of treatment. Total body and regional images of the upper limbs were acquired and interpreted by two nuclear medicine physicians blinded to the clinical findings. Joints showing focally increased tracer uptake compared to the background were considered positive and joints showing uptake equal to or less than background were considered negative.
Data of 30 patients were used for inter-observer and inter-modality agreement calculations. Changes in PET parameters and DAS28 were compared in 27 patients to assess treatment response.

Results: Out of 1560 joints examined in the initial scan, 394 were positive on PET/CT compared to 348 on clinical evaluation. Inter-observer agreement between nuclear medicine physicians was excellent (Cohen's kappa 0.92 $\mathrm{p}<0.05)$ and inter-modality agreement between PET and clinical examination was moderate (Cohen's kappa 0.55, p<0.05). The DAS28 and SUVmax values (highest and average) of 27 patients showed significant reduction on follow-up compared to the initial evaluation. There was significant correlation between percentage change in DAS28 and percentage change in scan parameters like PET positive joint counts $(0.689, p<0.001)$, average SUVmax $(0.712$ $p<0.001)$ and highest SUVmax values $(0.558, p=0.003)$ of scan-positive joints in 27 patients. Additional advantages of ${ }^{68} \mathrm{Ga}-\mathrm{RGD}, \mathrm{PET} / \mathrm{CT}$ included objective assessment, whole body evaluation of all small and large joints, and greater reproducibility.

Conclusion: ${ }^{68} \mathrm{Ga}-\mathrm{RGD}, \mathrm{PET} / \mathrm{CT}$ is a promising tool for objective assessment of disease activity and treatment response in patients with RA.

Table 1. Clinical and PET parameters of the patients

\begin{tabular}{lcc}
\hline Parameter & $\begin{array}{c}\text { Initial data }(n=27) \\
\text { Mean (SD)/Median (IQR) }\end{array}$ & $\begin{array}{c}\text { Follow-up data }(n=27) \\
\text { Mean (SD)/Median (IQR) }\end{array}$ \\
\hline TJC(28) & $10(5-13)$ & $3(2-4)$ \\
SJC(28) & $6(3-7)$ & $1(0-2)$ \\
ESR & $25(20-41)$ & $24(18-35)$ \\
PtGA & $6.0(5.0-6.0)$ & $3.0(2.0-4.0)$ \\
DAS28(3) & $5.14(0.85)$ & $3.74(0.88)$ \\
DAS28(4) & $5.60(0.90)$ & $3.80(0.96)$ \\
PET positive Joints & $12(7-8)$ & $4(2-9)$ \\
aSUVmax & $2.08(1.68-2.52)$ & $1.79(1.00-2.06)$ \\
hSUVmax & $3.45(2.71-4.70)$ & $3.34(1.95-4.25)$ \\
\hline
\end{tabular}

TJC/SJC: tender/swollen joint counts; ESR: erythrocyte sedimentation rate; PtGA: patients global assessment scale; DAS: disease activity score; aSUVmax/hSUVmax: average/highest SUVmax (maximum standardized uptake value); SD: standard deviation; IQR: interquartile range

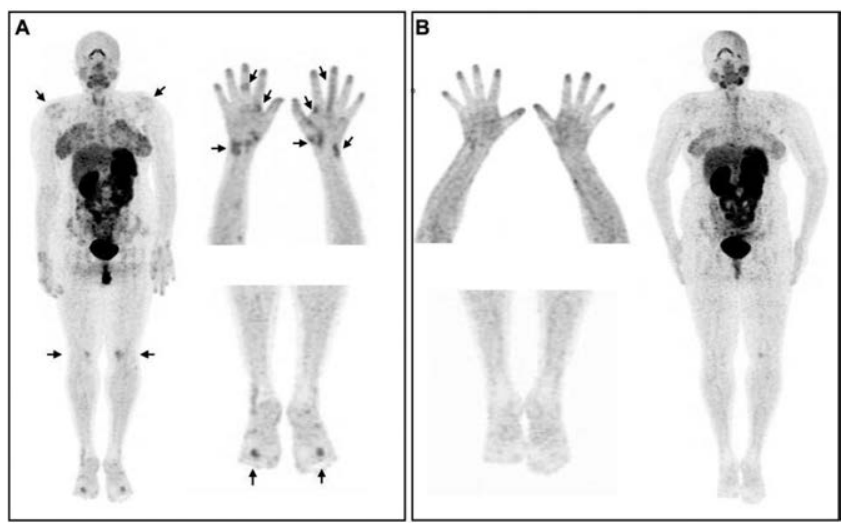

Figure 1. ${ }^{68} \mathrm{Ga}-\mathrm{RGD}$ PET scan of a 26-year female RA patient on treatment. A. Initial scan shows increased tracer uptake in multiple joints of upper and lower limbs and tendon sheaths of hands and ankle region (arrows); DAS28 was 4.56 (moderate disease activity) and ESR $12 \mathrm{~mm} / 1^{\text {st }}$ hour. B. Follow-up scan after 4 months shows resolution of tracer activity in the previously involved joints with only a mild focus persisting in the left knee joint; DAS28 was 1.73 (clinical remission according to ARA) and ESR $08 \mathrm{~mm} / 1^{\text {st }}$ hour.

Acknowledgments: This study was supported by Indian Council of Medical Research, New Delhi [grant no.3/2/June-2017/PG-Thesis-HRD (23)]

Disclosure of Interests: None declared

DOI: 10.1136/annrheumdis-2020-eular.3276

\section{THU0532 SCINTIGRAPHY TO PREDICT SPINAL PROGRESSION IN EARLY AXIAL SPONDYLOARTHRITIS: A PILOT STUDY}

S. H. Kim ${ }^{1}$, H. K. Min ${ }^{1}$, H. R. Kim ${ }^{1}$, S. H. Lee ${ }^{1}{ }^{1}$ Konkuk University Medical Center, Seoul, Korea, Rep. of (South Korea)

Background: Axial spondyloarthritis (axSpA) is a chronic inflammatory disease that typically affects the axial joint and enthesis. Abnormal hyperplasia of osteoblasts in the vertebral corner is the underlying pathogenesis of syndesmophyte 